\title{
Correction: Kikuchi, K., et al., Potential of the Angiotensin Receptor Blockers (ARBs) Telmisartan, Irbesartan, and Candesartan for Inhibiting the HMGB1/RAGE Axis in Prevention and Acute Treatment of Stroke. Int. J. Mol. Sci. 2013, 14, 18899-18924.
}

Kiyoshi Kikuchi ${ }^{1,2,3}$, Salunya Tancharoen ${ }^{1}$, Takashi Ito ${ }^{4}$, Yoko Morimoto-Yamashita ${ }^{5}$, Naoki Miura ${ }^{6}$, Ko-ichi Kawahara ${ }^{7}$, Ikuro Maruyama ${ }^{4}$, Yoshinaka Murai ${ }^{2}$ and Eiichiro Tanaka ${ }^{2, *}$

1 Department of Pharmacology, Faculty of Dentistry, Mahidol University, 6 Yothe Road, Rajthevee, Bangkok 10400, Thailand; E-Mails: kikuchi_kiyoshi@kurume-u.ac.jp (K.K.); salunya.tan@mahidol.ac.th (S.T.)

2 Division of Brain Science, Department of Physiology, Kurume University School of Medicine, 67 Asahi-machi, Kurume 830-0011, Japan; E-Mail: ymurai@med.kurume-u.ac.jp

3 Department of Neurosurgery, Kurume University School of Medicine, 67 Asahi-machi, Kurume 830-0011, Japan

4 Department of Systems Biology in Thromboregulation, Kagoshima University Graduate School of Medical and Dental Sciences, 8-35-1 Sakuragaoka, Kagoshima 890-8520, Japan;

E-Mails: takashi@m3.kufm.kagoshima-u.ac.jp (T.I.); rinken@m3.kufm.kagoshima-u.ac.jp (I.M.)

5 Department of Restorative Dentistry and Endodontology, Kagoshima University Graduate School of Medical and Dental Sciences, 8-35-1 Sakuragaoka, Kagoshima 890-8544, Japan;

E-Mail: yokomaru@dent.kagoshima-u.ac.jp

6 Laboratory of Diagnostic Imaging, Department of Veterinary Science, Faculty of Agriculture, Kagoshima University, 1-21-24 Korimoto, Kagoshima 890-0065, Japan;

E-Mail:nm18@vet.kagoshima-u.ac.jp

7 Laboratory of Functional Foods, Department of Biomedical Engineering Osaka Institute of Technology, 5-16-1 Omiya, Asahi Ward, Osaka 535-8585, Japan; E-Mail: kawahara@bme.oit.ac.jp

* Author to whom correspondence should be addressed; E-Mail: eacht@med.kurume-u.ac.jp; Tel.: +81-942-31-7542; Fax: +81-942-31-7695. 
The original version of the paper [1] reports that "This ACTIVE I study was supported by Pfizer" (Page 18905). However, the sponsors of the ACTIVE I study were actually Bristol-Myers Squibb and Sanofi-Aventis rather than Pfizer. Therefore, we would like to correct the wording to read: “This ACTIVE I study was supported by Bristol-Myers Squibb and Sanofi-Aventis". The authors apologize for any inconvenience this may have caused to the readers of this journal.

\section{Reference}

1. Kikuchi, K.; Tancharoen, S.; Ito, T.; Morimoto-Yamashita, Y.; Miura, N.; Kawahara, K.-I.; Maruyama, I.; Murai, Y.; Tanaka, E. Potential of the Angiotensin Receptor Blockers (ARBs) Telmisartan, Irbesartan, and Candesartan for Inhibiting the HMGB1/RAGE Axis in Prevention and Acute Treatment of Stroke. Int. J. Mol. Sci. 2013, 14, 18899-18924.

(C) 2014 by the authors; licensee MDPI, Basel, Switzerland. This article is an open access article distributed under the terms and conditions of the Creative Commons Attribution license (http://creativecommons.org/licenses/by/3.0/). 